\title{
La réorganisation des échanges internationaux de produits agricoles
}

\author{
Quelles perspectives pour l'agriculture européenne?
}

Philippe DUSSER

FOP

12 avenue George $V$

75008 Paris

$<$ p.dusser@prolea.com>

\begin{abstract}
Markets for agricultural commodities are changing patterns: the leadership is moving between commodities as well as between countries. Demands for meals for feed as well as demand for vegetable oil for human consumption have resulted in a strongly growing world market for oilseed which are seriously challenging the leadership to cereals in term of volume (170 million tons vs 200 million tons) while already coming first in value ( $\$ 45$ billion vs $\$ 25$ billion). The growth in demand is mainly localized in emerging countries in Asia (China, India) due to rising consumption of white meats: poultry and pork. Countries tend to produce locally the meats and the grains for feed, while accepting to rely strongly on imports for additional needs of proteins for feed and as well as of vegetable oil for human consumption. This is sustaining the growth of oilseed world exchanges. Parallel to new import markets in emerging countries in Asia, new producers are taking the leadership in exports, outpacing the USA and $E U$, longtime leaders on ag. markets. South America, and Brazil in particular, thanks to land availability and cheap labor are progressively becoming leaders in soybean, pork, poultry, beef, orange juice, sugar... Russia, Ukraine, are resuming a significant presence on grains markets. For the European Union, the new world context leads to a serious review of future positioning, especially in light of the future WTO obligations - less protection on access, elimination of export subsidies and less internal support. EU Animal productions sector will suffer most from the new context. Grains and oilseed EU production will continue to increase. As prices for grains will be curbed to world market level, some additional land may be diverted to oilseed. Nevertheless the availabilities of cereals will maintain high EU export. Biofuels appear to be a strategic outlet for European agricultural sector, biofuels use grains and sugar beet for ethanol as well as oilseed for biodiesel. Extent of biofuels development will determine the final balance of EU arable crops and especially availability of grains to export.
\end{abstract}

Key words: agricultural commodities markets, oilseeds, European agriculture outlook, grains

Alors que la libéralisation de la PAC s'étend et s'approfondit progressivement, que la perspective de la disparition définitive des subventions à l'exportation, actée à l'OMC dans l'accord-cadre de juillet 2004, conduit à engager d'urgence une réflexion stratégique sur le positionnement de l'agriculture française à l'exportation, il semble utile de faire un point sur les évolutions récentes des marchés internationaux de commodités agricoles et les tendances futures.

\section{Une demande mondiale en forte croissance}

Première constatation, la demande mondiale est considérable : 6 milliards d'êtres humains aujourd'hui, 4 de plus d'ici 2050 selon les projections de la $\mathrm{FAO}$; la croissance vigoureuse observée dans le passé récent ne faiblira pas. Les réserves de progrès technique doivent permettre à la production d'y répondre comme cela a été le cas lors des 50 dernières années. Ceci implique également une progression considérable du commerce international.

Sur la poursuite de ce qui se passe actuellement, la croissance de la demande mondiale sera le fait de pays émergents, essentiellement en Asie.
Dans ces pays, il reste de grandes marges d'intensification de l'agriculture qui permettent d'envisager un développement de la production locale. Cependant, avec des surfaces agricoles plutôt en régression du fait de l'urbanisation, le recours à l'importation sera de toute évidence un des éléments de réponse à la satisfaction d'une consommation en forte croissance quantitative et... qualitative, c'est-àdire avec moins de céréales consommées directement (blé, riz) et davantage de viandes et de matières grasses.

Protéines et huiles végétales, stars des marchés internationaux par la grâce des viandes blanches

La consommation de viandes blanches, porc et volaille, connaît une forte croissance (figure 1). La production mondiale est en hausse de 3 à $4 \%$ par an (en 2004, elle atteint 90 millions de tonnes de porc et 60 millions de tonnes de volaille) (figure 2).

Les échanges de ces viandes (4 millions de tonnes de porc et 7 millions de tonnes de volailles) progressent annuellement de $5 \%$ (figure 3).

Ces productions de viande blanche en croissance ont des effets considérables sur les échanges de produits végétaux, elles nécessitent en effet de grandes quantités d'aliment : 3 à $4 \mathrm{~kg}$ par $\mathrm{kg}$ de viande, dont 70 à $75 \%$ de céréales et 20 à $25 \%$ de tourteau protéique.

C'est la demande pour l'alimentation animale qui tire depuis 10 ans les échanges de produits végétaux.

Cependant, déjouant les prévisions, ce ne sont pas les échanges de céréales qui en ont bénéficié, mais ceux d'oléagineux.

Ces derniers, ont par ailleurs le double intérêt de répondre à la fois aux besoins d'alimentation animale et à la demande de matières grasses pour l'alimentation humaine également en pleine croissance (figure 4).

Le soja domine le marché des tourteaux, mais I'huile de soja partage également la première place à égalité avec l'huile de palme. Les autres oléagineux, colza, tournesol, graine de coton, arachide arrivent assez loin derrière.

La croissance de la production et des échanges d'oléagineux, au cours de ces dernières années est remarquable (figure 5). Les échanges d'oléagineux en particulier (figure 6) sont en passe de ravir la première place aux céréales: en valeur c'est déjà le cas depuis plusieurs années (40 milliards de dollars pour les oléagi- 


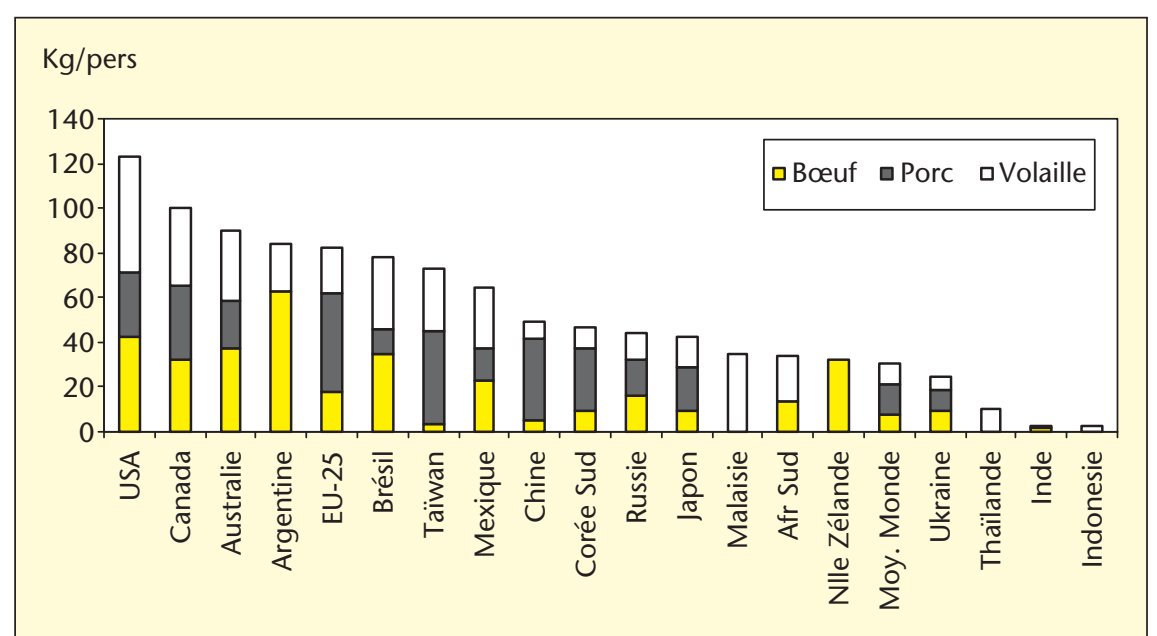

Figure 1. Consommation mondiale de viande par habitant en 2004.

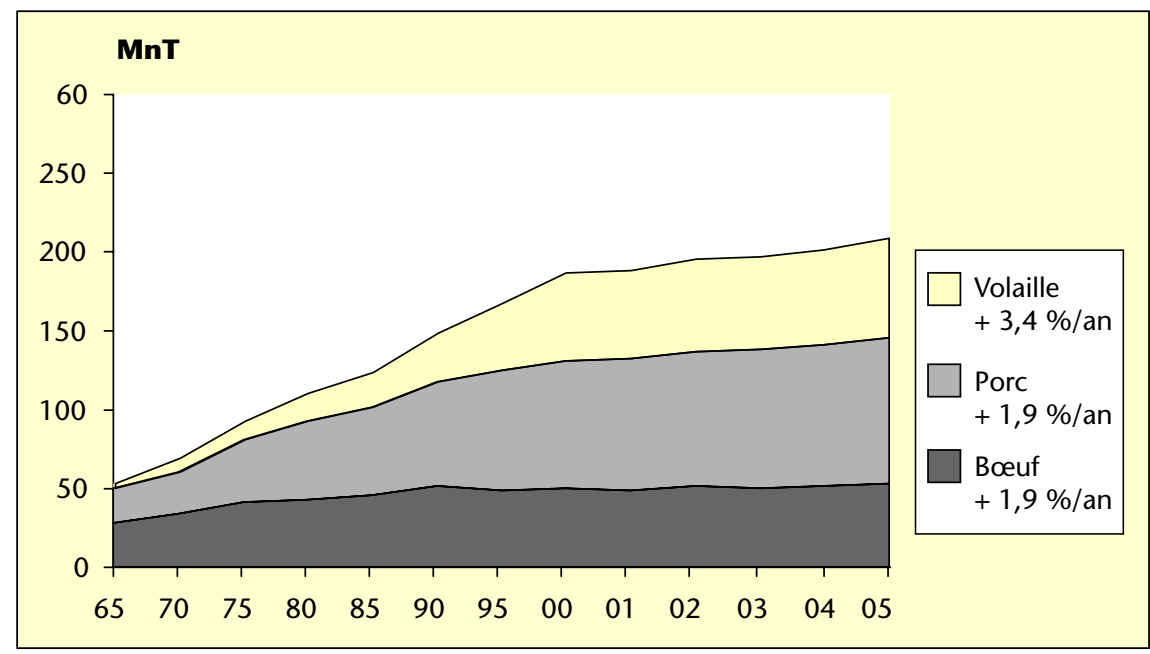

Figure 2. Production mondiale de viandes.

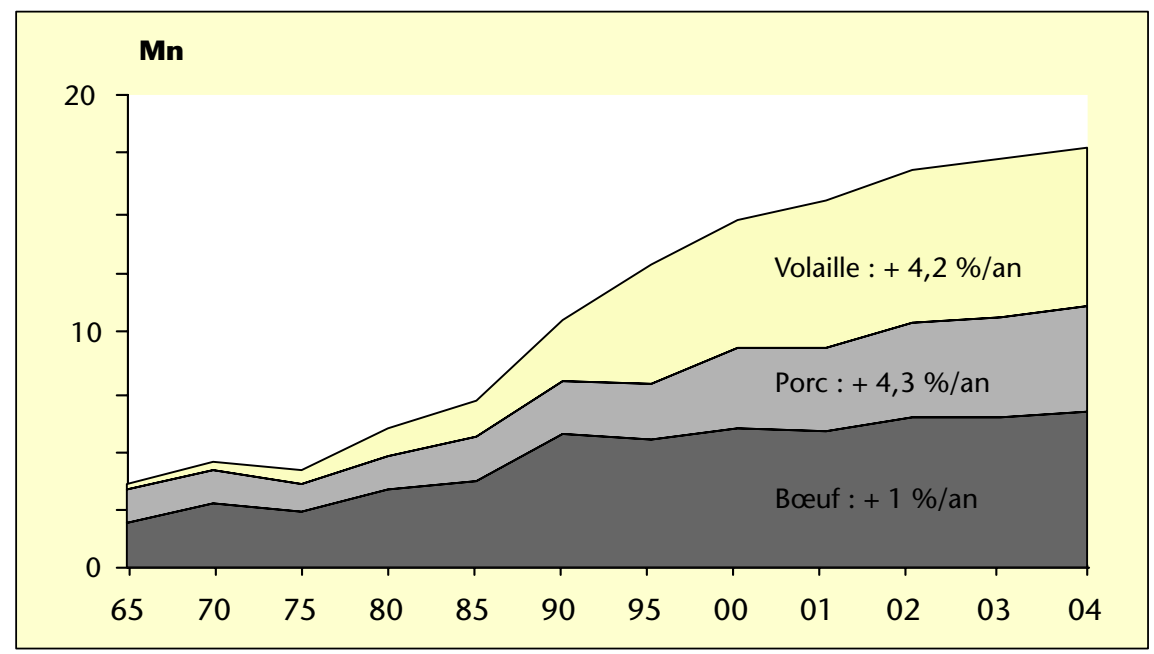

Figure 3. Echanges mondiaux. neux, 25 milliards de dollars pour les céréales), mais bientôt ce sera le cas en volume (figure 7). Quelques chiffres : en 10 ans, la production de céréales a augmenté de $1 \%$ par an (soit 400 millions de tonnes supplémentaires), celle d'oléagineux de $4 \%$ par an (280 millions de tonnes supplémentaires). Les échanges de céréales dans le même temps stagnaient à 200 millions de tonnes (100 pour le blé et 100 pour les céréales secondaires) pendant que ceux concernant les oléagineux passaient de moins de 100 à plus de 170 millions de tonnes (dont 110 de tourteaux protéiques et 60 d'huiles).

Les échanges de céréales vont-ils décoller?

Est-ce à dire que ce n'est que partie remise pour les céréales ? Peut-être la demande de matière première pour I'alimentation animale va-t-elle finalement déboucher sur l'augmentation attendue des échanges.

Néanmoins, les politiques de sécurité alimentaire mises en place dans les pays comme l'Inde ou la Chine jouent un rôle non négligeable en la matière. Jusqu'à maintenant, on a observé que ces pays ont systématiquement privilégié l'encouragement de leur propre production de céréales, alors qu'ils acceptaient de dépendre à un degré important des importations pour les protéines végétales et les huiles.

En témoignent, en Inde, la révolution verte qui a amené ce pays à une indépendance en céréales depuis les années 50, et en Chine, une politique conduisant la constitution d'énormes stocks de céréales au cours des années 90 . Parallèlement, ces deux pays sont devenus les plus gros importateurs d'oléagineux (huile pour l'Inde, graines de soja pour la Chine).

\section{Importations : I'Asie, et principalement la Chine, tire les marchés}

\section{La Chine}

Dans le domaine des échanges de produits agricoles comme ailleurs, la Chine est la clé des évolutions récentes et futures. Mais il convient d'être prudent : si la croissance de la consommation est bien là, en matière d'importation, les prévisions ont souvent été démenties par la réalité.

Le rapport très particulier entretenu par l'administration chinoise avec les statistiques complique très largement un exercice par nature difficile dans un pays qui reste encore très marqué par des habitudes de politiques économiques dirigistes.

La consommation de viande augmente régulièrement, satisfaite pour le moment essentiellement par la production locale : la production 


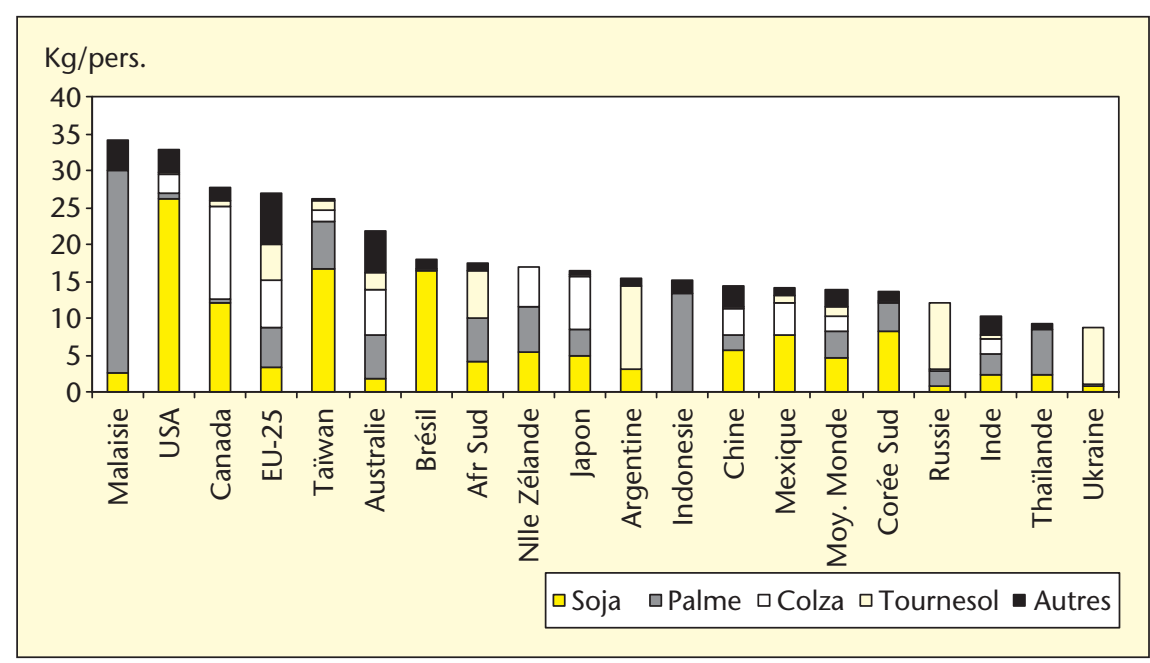

Figure 4. Consommation mondiale d'huile végétale par habitant.

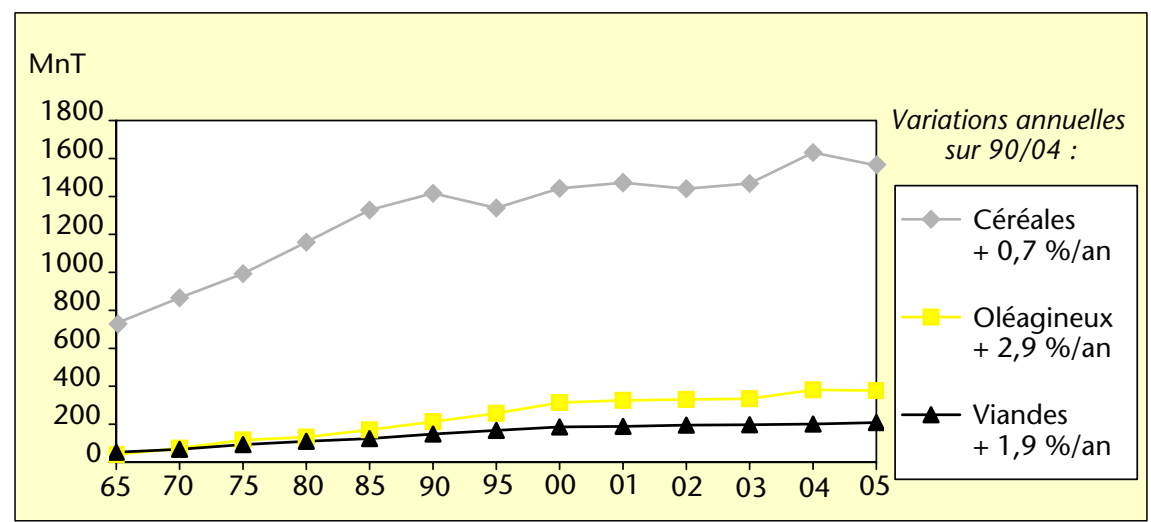

Figure 5. Productions mondiales d'oléagineux, de céréales et de viandes (tonnage).

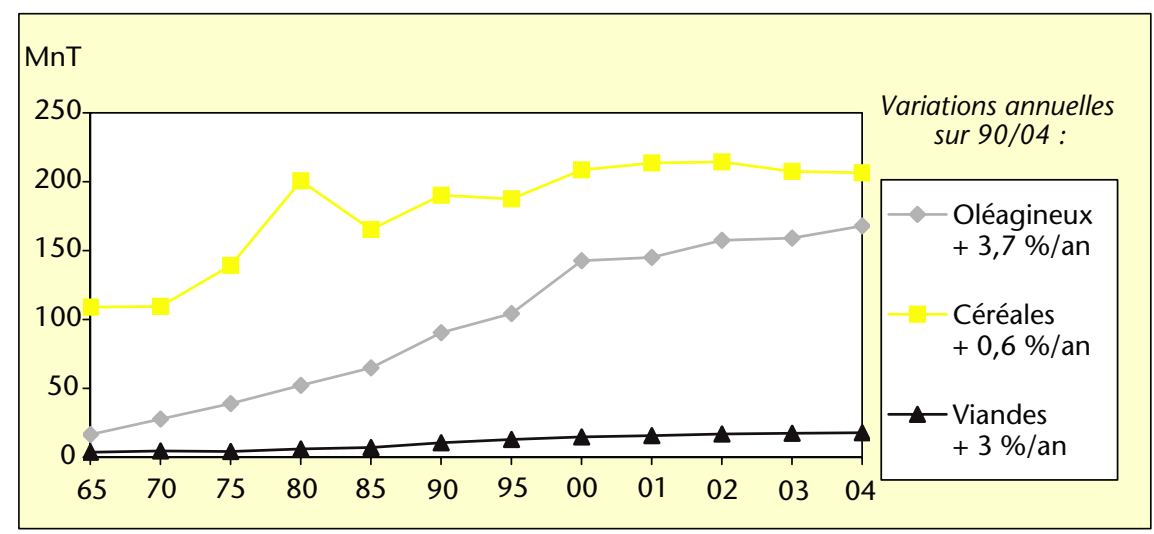

Figure 6. Échanges mondiaux d'oléagineux, de céréales et de viandes (tonnage).

de porc (50 millions de tonnes) augmente de $4 \%$ par an, celle de volailles (10 millions de tonnes) de $6 \%$ par an (figure 8 ).

La production chinoise de viande blanche est pour l'essentiel produite avec des céréales locales avec en complément des quantités chaque année plus importantes de tourteau de soja importé. vues cette année (figure 9). L'explication de cette disparition : à l'insu de toute statistique, des stocks considérables s'étaient accumulés en réponse à une politique de prix incitatifs. Ces stocks ont fini par atteindre environ l'équivalent d'un an de consommation soit environ 300 à 400 millions de tonnes de riz, blé et maïs, avant qu'une nouvelle politique $n^{\prime}$ induise une baisse importante de la production, et leur résorption progressive. Ils arriveraient maintenant à un niveau d'équilibre.

Le cas du maïs est particulier ; traditionnellement, la Chine, exporte un peu de maïs : 5 millions de tonnes, cette situation jusqu'à maintenant a peu évolué mais devrait s'inverser dans le futur.

C'est dans le secteur oléagineux qu'a eu lieu l'explosion des importations attendue ailleurs. Très gros producteur d'oléagineux (soja et colza principalement), la Chine jusqu'en 1990 était plutôt en position d'exportateur.

En 10 ans, les importations d'oléagineux essentiellement soja - ont progressé pour atteindre 25 millions de tonnes en 2004 ; la part de marché de la Chine, en augmentation constante, va bientôt atteindre $20 \%$ et finira bien par rattraper celle de l'Union européenne qui avec 50 millions de tonnes reste encore le premier importateur mondial (figure 10).

Si, au début, les Chinois importaient d'un côté des tourteaux de soja des États-Unis et d'Amérique du sud, et de l'autre de l'huile de palme $d^{\prime}$ Indonésie et Malaisie, ils ont rapidement décidé d'importer des graines de soja qu'ils transforment eux-mêmes pour en tirer I'huile et les tourteaux.

Pour le futur, il semble bien que les dirigeants chinois envisagent une croissance contrôlée des importations de céréales, et la poursuite de la forte progression de la tendance actuelle pour les oléagineux. En témoignent les engagements pris par la Chine à l'OMC : un contingent de 22 millions de tonnes pour les céréales (10 de blé, 7 de maïs, 5 de riz) et un accès libre pour les oléagineux.

\section{Dans le reste de l'Asie}

Des pays émergents comme les Philippines, I'Indonésie, la Malaisie, représentent des marchés en expansion pour les céréales (25 millions de tonnes dont 15 de blé soit 5 de plus en 10 ans), mais également pour les tourteaux oléagineux, et la viande.

Certains pays sont également des producteurs et des exportateurs, de premier plan : Indonésie et Malaisie sont leaders dans les exportations d'huile de palme.

La Thaillande exporte du maïs, de la volaille, des produits de la mer à destination de pays de la zone mais aussi sur les marchés européens.

Du côté des pays développés ou avancés, Japon, Corée du Sud, Taïwan représentent 


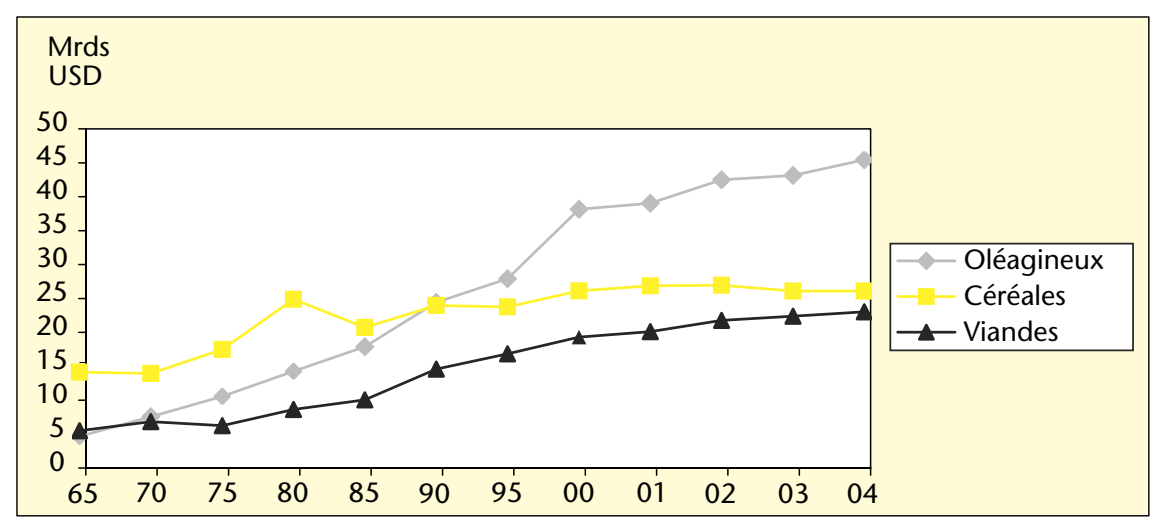

Figure 7. Valeur des échanges mondiaux d'oléagineux, de céréales et de viandes - (Base prix moyens 94-04).

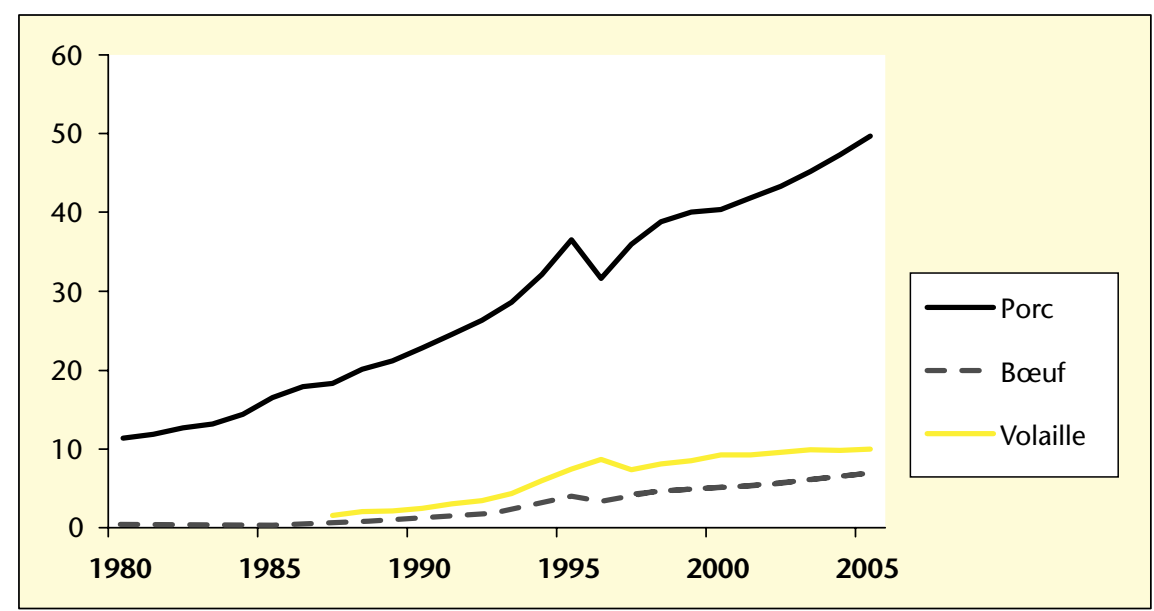

Figure 8. Chine: production de viandes (1 000 TEC).

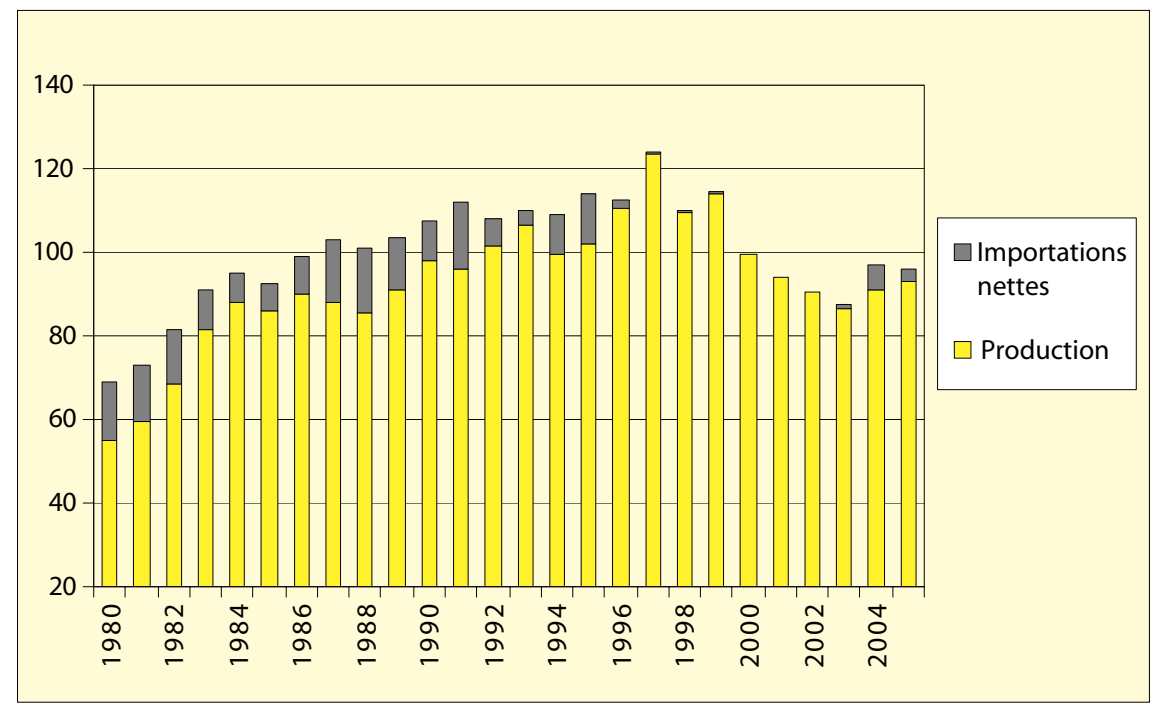

Figure 9. Chine : production et importations de blé (MnT).

$20 \%$ du marché des céréales pour des volumes stationnaires, leurs importations de viandes sont quant à elles en augmentation forte : $+40 \%$ en 10 ans.
L'Inde occupe une place à part : très protectionniste, sa révolution verte lui a permis de rester autosuffisante en céréales jusqu'à maintenant. Les habitudes végétariennes excluent une expansion importante des viandes, encore que sur la volaille il existe un potentiel. Par contre, I'Inde est le premier importateur mondial d'huile, ainsi que de légumes secs. Une révolution « jaune » destinée à encourager les productions d'oléagineux et de légumineuses lancée dans les années 1990 n'a pas atteint I'objectif d'auto-suffisance. L'Inde restera manifestement dépendante des importations pour ces produits.

\section{Exportateurs : la montée en puissance de l'Amérique du Sud}

\section{Les États-Unis sont en train de perdre le leadership}

De nouveaux acteurs, relativement secondaires voire inexistants il y a encore 10 ans, sont en train de prendre rapidement les premières places sur des marchés mondiaux des viandes, des oléagineux, et peut-être celui des céréales.

- l'Amérique du Sud vient de conquérir la première place sur les marchés du soja et du sucre, elle poursuit sa percée sur ceux de la volaille, du porc, du bœuf. Les ambitions affichées sont à la hauteur du potentiel - très élevées - et surtout désormais ciblées sur les produits à valeur ajoutée.

- Quelques pays asiatiques tirent parti de leurs atouts: la Thaillande développe des productions animales d'exportation (volailles, produits de la pisciculture), la Malaisie et l'Indonésie dominent le marché des huiles avec leurs exportations d'huile de palme ;

- Pas encore fermement installés sur les marchés internationaux, on voit bien que les pays de la Mer noire (Russie, Ukraine) vont reprendre à échéance assez courte la place qu'ils avaient au XIX ${ }^{\mathrm{e}}$ siècle dans le commerce des grains - céréales et oléagineux.

\section{L'Amérique du Sud, en passe de dominer les marchés agricoles}

Le phénomène le plus marquant est sans conteste la montée en puissance de l'Amérique du Sud.

Elle connaît actuellement un extraordinaire boom des exportations de produits agricoles. On peut en particulier y observer une véritable fièvre du soja à peu près généralisée à tous les pays du cône sud.

Les situations sont néanmoins relativement diverses suivant les pays.

\section{L'Argentine}

Traditionnellement tournée vers l'exportation, continue à affirmer sa présence sur les marchés mondiaux du blé, du soja, du tournesol et de la viande bovine. Ce qui frappe ces dernières 


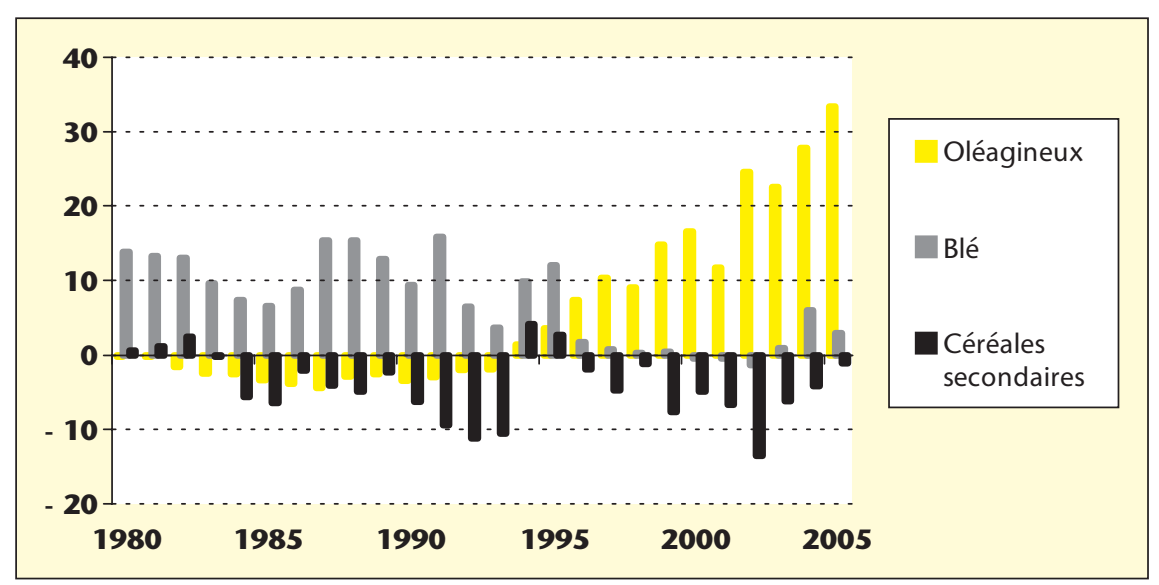

Figure 10. Chine : importations nettes de céréales et d'oléagineux (MT).

années c'est l'intensification de l'agriculture argentine: les terres consacrées à l'élevage régressent, et sont mises en culture pour la production de soja. La production de viande bovine ne baisse pas pour autant car l'élevage s'intensifie.

\section{Paraguay, Bolivie, Uruguay}

Ils sont également gagnés par la fièvre du soja. Des territoires importants sont défrichés, mis en culture, la production dirigée pour l'exportation vers les ports de l'Atlantique : Rosario en Argentine ou Paranagua au Brésil. Les acteurs de cette expansion sont très souvent des Argentins ou des Brésiliens, mais également des investisseurs venant de plus loin, des ÉtatsUnis en particulier.

\section{Le Brésil, géant agricole encore en phase de croissance}

Le Brésil avec son potentiel extraordinaire domine de loin les perspectives de développement de la zone.

Traditionnellement davantage centré sur son important marché intérieur, le Brésil s'est tourné depuis 10 ans vers le marché mondial, et le résultat est impressionnant. Habituellement en position de tête dans les produits tropicaux, jus d'orange, café, le Brésil a conquis en quelques années les premières places sur les marchés mondiaux du sucre, du soja, de la viande bovine, des viandes blanches - porc et surtout volaille - il s'intéresse actuellement à d'autres secteurs comme celui du coton. Le Brésil dispose de très forts atouts : pays continent avec des territoires immenses encore à défricher, une population nombreuse, à la fois main-d'œuvre bon marché et base d'un important marché intérieur, une classe moyenne et supérieure très bien formée et éduquée, à même d'encadrer le développement économique.

- Le soja règne sur les nouvelles zones de culture du centre et de l'Amazonie.
Le Brésil, c'est surtout la dernière frontière agricole du monde où année après année dans les « cerrados » du centre-ouest, la savane arborée fait place au soja sur des exploitations de plusieurs milliers d'hectares.

Le soja est en effet la plante pionnière de cette zone, et son développement a permis à la production brésilienne de passer en une dizaine d'années de 25 à 60 millions de tonnes, dont plus de 40 millions de tonnes exportées ont délogé les Etats-Unis de la place de premier exportateur (figures 11 et 12).

Sur cette frontière, $s^{\prime}$ installent progressivement d'autres productions comme le coton, mais également l'élevage bovin et des ateliers de

Le potentiel reste considérable : le gouvernement brésilien évalue à 90 millions d'hectares la réserve de terres à mettre en culture dans les zones de savanes (théoriquement sans toucher à la forêt équatoriale). À titre de comparaison, il viande blanche.

Figure 11. Brésil : production de grains (MT). est intéressant de noter que cette surface est supérieure à la totalité des terres arables de I'Union européenne à 25 (85 millions d'hectares) et représente plus que la moitié de celles des États-Unis (170 millions d'hectares).

Dans cette zone, la question des moyens de transport est cependant cruciale : elle est loin d'être résolue correctement, et c'est par camion sur des milliers de kilomètres de routes pas toujours goudronnées que le soja rejoint les ports de Paranagua sur l'Atlantique ou les barges qui I'achemineront à Santarem sur l'Amazone.

- Le sucre continue son développement dans la région de Sao Paulo.

Le sucre est également un des secteurs que le Brésil est en passe de dominer au niveau mondial.

La production de canne à sucre est localisée principalement dans la région de Sao Paulo sur les anciennes zones de production du café. Cette production connaît un grand développement depuis plus de 30 ans. Liée à la production d'éthanol pour le carburant après la crise pétrolière de 73 , réorientée dans les années 1990 vers l'exportation de sucre sur le marché mondial, cette production a été relancée récemment pour la production d'éthanol carburant destiné au marché intérieur... ainsi qu'à l'exportation (I'UE a proposé un contingent de 1 million de tonnes dans l'offre d'accord commercial avec le Mercosur).

En dix ans la production de sucre a doublé pour atteindre 24 millions de tonnes, et avec une consommation qui reste stable, les exportations atteignent aujourd'hui 14 millions de tonnes, soit $35 \%$ du marché mondial.

- Viandes blanches: les ateliers des États du Sud à la conquête du monde (figure 13).

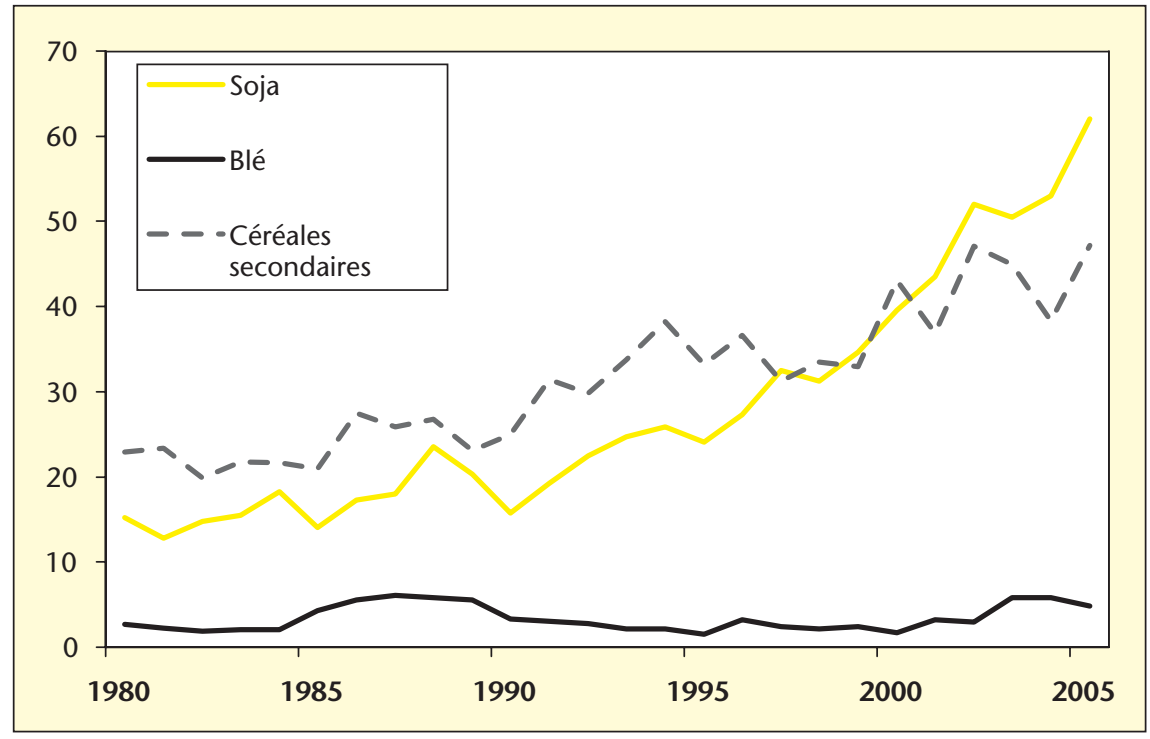




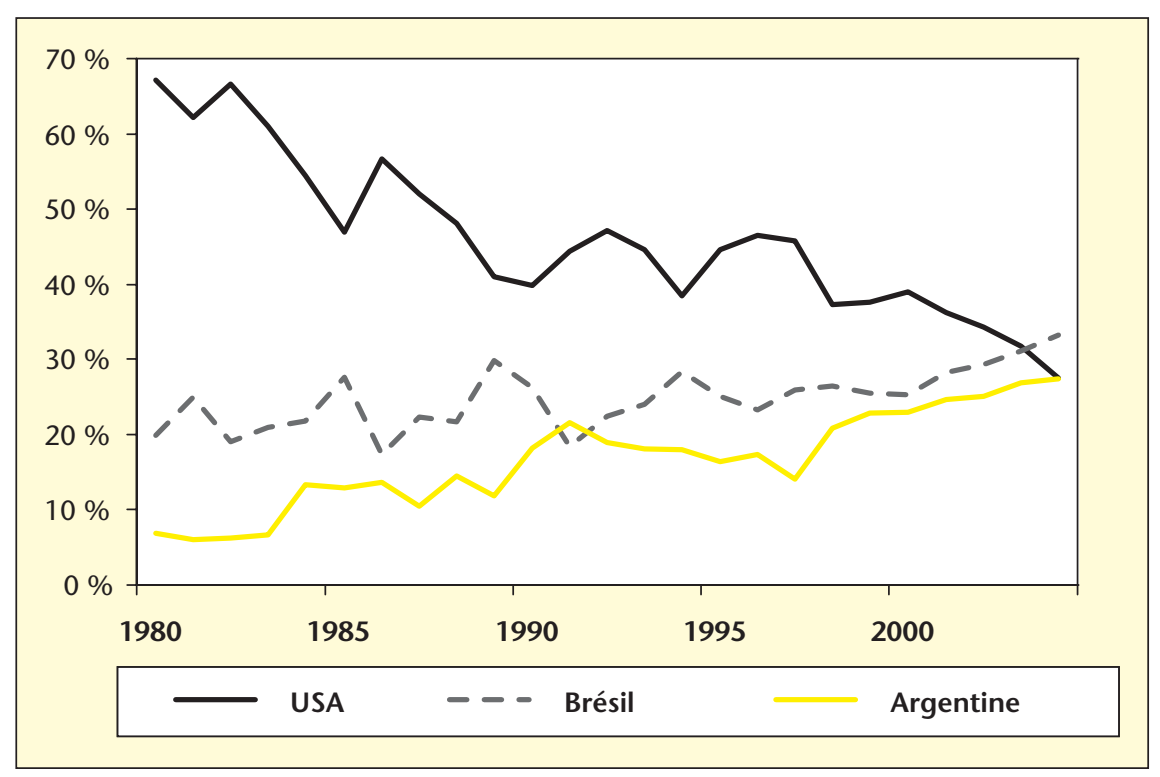

Figure 12. Exportations de soja (graines + huile + tourteaux) en \% du total mondial.

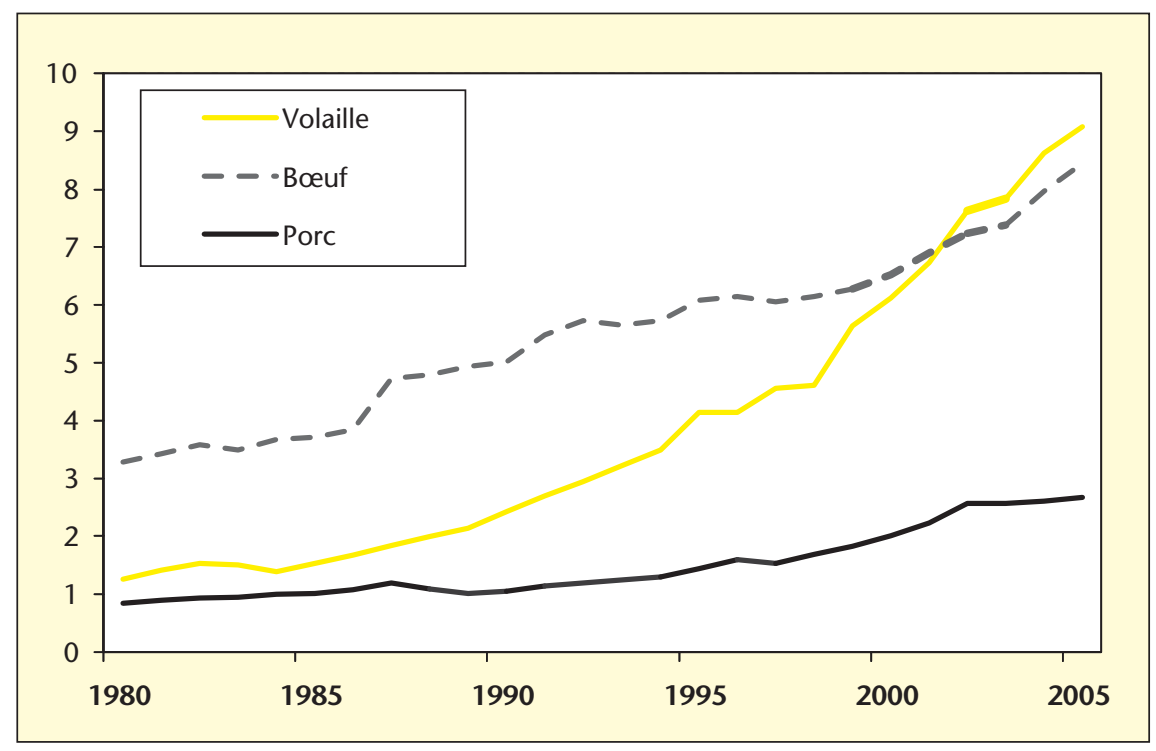

Figure 13. Brésil : production de viandes (M TEC).

Dans la viande blanche enfin, la percée du Brésil est spectaculaire, en volaille particulièrement. En quelques années, les États-Unis ont été détrônés de leur position de leader, et I'Union européenne voit sa production mise en danger non seulement sur les marchés $d^{\prime}$ exportation mais également sur son propre marché intérieur du fait d'importations qui se jouent des protections à l'accès.

Ces productions sont concentrées dans les zones du sud-est du Brésil (Rio Grande do Sul, Santa Catarina) où une population traditionnelle de petits agriculteurs est à même de transformer les céréales et le soja brésilien en porc et en volaille à des coûts inférieurs de moitié à ceux de leurs homologues des États-Unis ou ont doublé pour atteindre plus d'un million de tonnes, et amener le Brésil pratiquement au niveau de l'Australie, premier exportateur (figure 14).

- Le Brésil attire les investissements internationaux.

Dans tous les secteurs, nous sommes en train d'assister à la naissance d'un géant agricole. Les investisseurs ne $s^{\prime} y$ trompent pas, des milliers $d$ 'hectares sont achetés par des étrangers, compagnies, fonds d'investissements, producteurs - américains le plus souvent. Les investisseurs chinois commencent à arriver, eux aussi, au Brésil.

Mais on voit également les entreprises européennes compétitives bloquées dans leur développement par les évolutions de la PAC s'installer là-bas pour retrouver un potentiel de croissance: c'est le cas de volaillers qui ne peuvent plus exporter pour cause de réduction des soutiens à l'exportation, c'est également le cas de sucriers, confrontés à la perspective de la réduction de la production européenne, dans le cadre de la réforme à venir (baisse des quotas de production et baisse des prix).

\section{Quelles perspectives pour l'Europe?}

Les évolutions du contexte mondial amènent à réfléchir sur le positionnement de l'UE: la concurrence mondiale va se trouver renforcée tandis que parallèlement, de grandes opportunités sont en train de se concrétiser, qu'il est possible et nécessaire de saisir.

Le résultat des négociations en cours à l'OMC est central pour les orientations futures qui seront ouvertes à l'agriculture européenne. La disparition des subventions à l'exportation, concession majeure de I'Union déjà actée à I'OMC, impliquera, à une échéance encore à négocier, des ajustements stratégiques et très probablement un recentrage sur le marché intérieur pour certains secteurs.

Le volet « accès » de la négociation reste par ailleurs encore totalement à mener : quelles réductions sur les droits de douane, quel traitement pour les produits sensibles? De la situation de la protection du marché intérieur à l'issue des négociations dépendront les possibilités de redéploiement.

Ces perspectives nécessitent surtout d'anticiper l'adaptation des structures de l'appareil de production et de transformation - des filières de production - au nouveau contexte européen et mondial qui se met en place.

Pour essayer de dégager des pistes, on peut se risquer à faire quelques hypothèses sur les différents secteurs et leurs évolutions.

\section{Les filières animales}

De fortes incertitudes pèsent sur ces secteurs. 


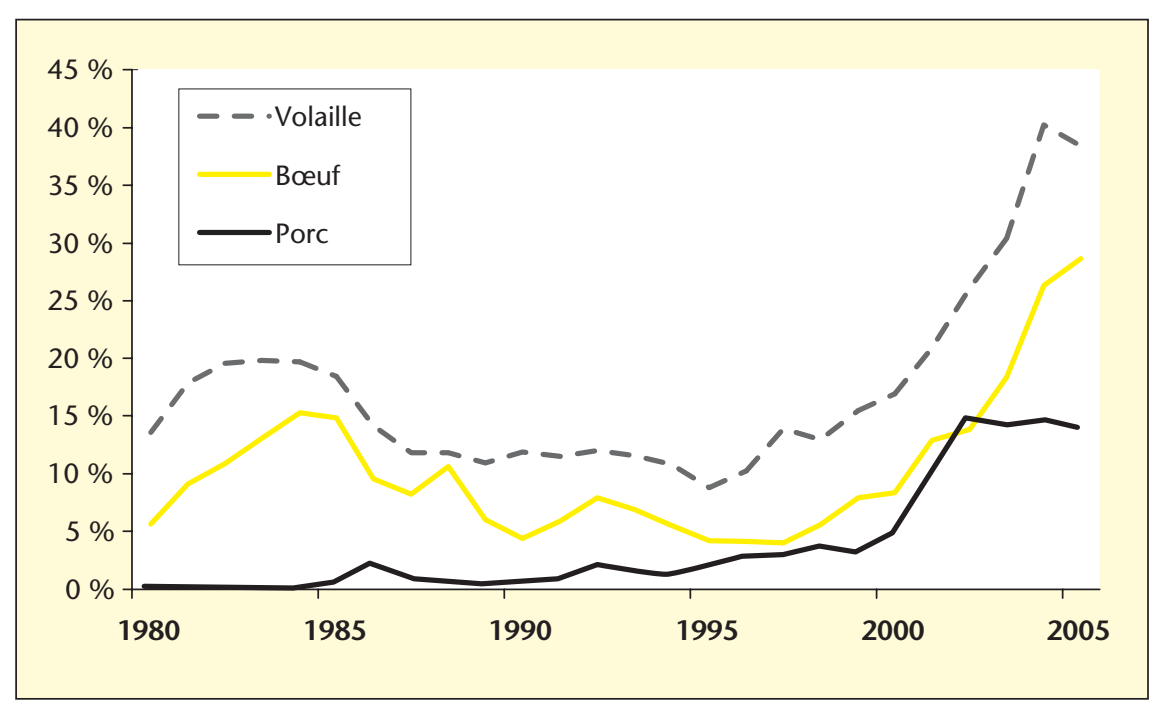

Figure 14. Brésil : part des exportations mondiales de viandes (\%).

Un repli sur le marché intérieur a déjà été amorcé à la suite des contraintes à l'exportation imposées par l'accord de Marrakech, qui a conclu en 1994 le dernier round de négociation de I'OMC.

À la faveur de brèches dans la protection communautaire (importations de volailles saumurées), ou plus simplement au travers de la progression de l'importation de produits semitransformés, la concurrence internationale commence par ailleurs à jouer dès à présent sur le marché communautaire.

Le résultat des futures négociations du Cycle de Doha accentuera les pressions de la concurrence internationale. Le dossier du traitement réservé aux produits sensibles et des possibilités de protection qu'en tirera l'Union européenne est capital pour nombre de ces filières.

Par ailleurs, suite à l'élargissement de I'Union européenne, s'engage un mouvement d'investissement dans les nouveaux États membres qui disposent de céréales difficiles à transporter, de coûts inférieurs pour l'infrastructure et la main-d'œuvre, et de demandes nationales en croissance, qui peut comporter des relocalisations pour certaines productions.

Une période de restructuration s'ouvre donc pour les secteurs animaux.

De la manière dont ces filières sauront s'adapter au nouveau contexte dépend également l'équilibre général du secteur des grandes cultures dont elles constituent le débouché principal. L'enjeu est donc collectif : c'est l'ensemble de l'agriculture qui est concerné par l'évolution de ces secteurs.

\section{Pour les grandes cultures}

Du côté des productions végétales, les choses se présentent différemment : en effet, la terre $n^{\prime}$ est pas délocalisable, et tant que les prix restent suffisants, les producteurs produisent.
Le risque de déprise agricole existe dans certaines régions moins productives, il reste cependant marginal, et la production de grandes cultures devrait continuer à progresser.

\section{Une baisse des prix des céréales entrainant des ajustements structurels}

Avec la fin programmée des restitutions à l'exportation, il faut anticiper une baisse des prix intérieurs des céréales; cette baisse des prix portera principalement sur les céréales secondaires pour lesquelles le différentiel entre marché mondial et prix intérieur est le plus important.

Pour maintenir leur compétitivité, les exploitations de grandes cultures devront engager des ajustements structurels et réduire leur coût de production par une baisse des charges. À cet égard, la poursuite de l'agrandissement des exploitations semble inévitable.

\section{Un redéploiement vers les oléoprotéagineux ? \\ C'est un pari que l'on peut faire.}

Une amélioration de l'intérêt économique pour les producteurs à s'orienter davantage vers les oléagineux résultera automatiquement du réaménagement des rapports de prix en leur faveur.

En effet, si pour les céréales on prévoit une baisse des prix intérieurs, ceux des oléagineux ne bougeront pas : ils sont depuis 15 ans directement au prix mondial.

De quelle ampleur pourrait être ce rééquilibrage? Pour donner un ordre de grandeur, on peut rappeler que la suppression en 2002 des soutiens spécifiques aux oléagineux qui compensaient depuis 1992 le handicap du prix mondial, avait entraîné une baisse des surfaces de $20 \%$ (1 million d'hectares).
En définitive, ce rééquilibrage dépendra de l'ampleur de la variation du rapport de prix entre céréales et oléagineux.

Sur ce point, on peut avancer deux hypothèses qui permettent de penser que les prix des oléagineux devraient rester soutenus relativement à ceux des céréales :

- une progression plus forte de la demande de protéines et d'huile : à I'horizon 2050, on anticipe une multiplication par 4 des besoins en oléoprotéagineux, contre une multiplication par 2 des besoins en céréales - cette différence s'expliquant par l'évolution des techniques d'alimentation animale dans les pays en développement.

- Les conséquences d'un scénario de hausse durable des coûts de fret. La pression à la baisse sur le prix départ des marchandises exportées et à la hausse sur celui des produits importés serait également un facteur de renforcement des prix relatifs des oléagineux à l'intérieur de I'Union européenne qui importe $75 \%$ de ses tourteaux d'oléagineux et $50 \%$ de ses huiles, mais continuera à exporter des céréales.

\section{Une augmentation des quantités de blé disponibles pour l'exportation... ou des usages non alimentaires}

Plusieurs facteurs incitent à prévoir des disponibilités supplémentaires de blé disponibles pour l'exportation :

- Tout d'abord la production de céréales devrait continuer à progresser.

Dans les anciens États membres, cette progression devrait suivre les tendances antérieures. Par contre dans les nouveaux États membres l'augmentation de la production devrait être forte: les marges d'intégration de progrès technique sont importantes, et les rendements sont encore très inférieurs à ceux du reste de l'Europe. Malgré une augmentation attendue de la demande intérieure de ces pays, ils devraient dégager des surplus exportables (on évoque la possibilité d'un surplus de 10 millions de tonnes).

- Parallèlement, I'utilisation des céréales dans I'alimentation animale a tendance à régresser ou tout du moins à stagner, suite aux difficultés des filières animales.

Cette tendance observée au niveau de l'ensemble de I'UE n'exclut pas un développement des productions animales dans les nouveaux États membres, soutenu à la fois par le développement de leur propre consommation, mais également par certaines relocalisations de production à partir des anciens États membres.

Hausse de la production, stagnation de la demande animale, ces perspectives laissent penser que l'Union européenne continuera à être un acteur majeur sur le marché mondial des céréales, et plus précisément du blé. 
Le contexte nouveau dans lequel ces exportations devront se faire (sans restitutions) rend $d^{\prime}$ autant plus nécessaire une organisation forte des outils de commercialisation à l'exportation, et en particulier un travail intense de promotion permettant de maintenir les parts de marchés sur les destinations traditionnelles et de pénétrer les marchés en développement.

\section{Les biocarburants : une opportunité à saisir}

Les biocarburants constituent bien évidemment une chance pour l'agriculture dont I'Union européenne doit se saisir.

Tout pousse à leur développement : le respect des objectifs de Kyoto pour la maîtrise des gaz à effet de serre tout d'abord, mais également désormais l'évolution du prix du pétrole. Tout indique aujourd'hui que la hausse des prix du pétrole devrait être durable: la demande d'énergie qui va augmenter de $50 \%$ dans les 50 ans à venir pour répondre aux besoins des pays émergents, la conscience du fait que les ressources en pétrole ne sont pas illimitées et la perception que le moment où ces limites commenceront à véritablement se faire sentir n'est éloigné que de quelques décennies; enfin, il n'est pas besoin de souligner l'instabilité politique des zones où sont situées ces réserves.

Les biocarburants, qui se sont développés jusqu'alors dans le cadre de décisions à caractère politique et $d$ 'incitations des pouvoirs publics avec des objectifs de sécurité d'approvisionnement et de respect de l'environnement, approchent désormais de beaucoup plus près le seuil de rentabilité économique. Au Brésil et aux États-Unis, ils n'en sont pas très loin.

Quelle sera leur place dans la production agricole? Quelle surface leur consacrer pour maintenir l'offre en alimentaire?

Des études commencent à être entreprises pour répondre à ces questions, signe que le dossier sort véritablement du statut marginal ou même expérimental qu'il avait jusqu'alors.

Au Brésil, l'éthanol (130 millions d'hectolitres en 2003) couvre $20 \%$ de la consommation totale de carburant, aux États-Unis cette part est environ de $10 \%$ (avec 100 millions d'hec- tolitres en 2003). Et ces parts devraient encore progresser dans ces deux pays.

Dans I'Union européenne, deux échéances ont été fixées : un objectif (indicatif) de 5,75\% des carburants pour les transports en 2010, et au-delà $20 \%$ d'énergies alternatives d'ici 2020.

Si l'objectif de 5,75\% était atteint à partir de productions locales, ceci pourrait représenter des surfaces et des productions importantes.

Il est actuellement trop tôt pour donner des chiffres sur l'impact des cultures de biocarburants sur les bilans de marché des différentes productions végétales: cela dépendra de la réalisation effective des objectifs par les pays, et de la manière dont ils les rempliront. Mais il est clair que cela aura un impact sur les soldes de céréales disponibles pour l'exportation, et également sur les prix et les surfaces d'oléagineux. Nouvelle frontière, les biocarburants représentent aujourd'hui une opportunité économique majeure mais également un enjeu de société qui peut donner l'occasion à l'agriculture européenne de renouveler son image. 